\title{
REPENSANDO LA TORRE DE MARFIL. REFLEXIONES SOBRE LA UNIVERSIDAD DE CHILE Y EL PROCESO CONSTITUYENTE
}

Davor Harasic Yaksic 


\section{DAVOR HARASIC YAKSIC}

Davor Harasic Yaksic es abogado y Licenciado en Ciencias Jurídicas y Sociales de la Universidad de Chile. Magíster en Derecho, mención en Derecho Penal por la Universidad de Chile. Es Profesor Titular y Decano de la Facultad de Derecho de la Universidad de Chile. 


\section{REPENSANDO LA TORRE DE MARFIL. REFLEXIONES SOBRE LA UNIVERSIDAD DE CHILE Y EL PROCESO CONSTITUYENTE}

\section{INTRODUCCIÓN}

Entre los meses de agosto y noviembre del año 2015, más de 60 académicos se encontraron para discutir en torno a la pregunta por una nueva Constitución Política para Chile. Fueron 15 las sesiones fecundas que formaron parte del Ciclo de Discusiones sobre Proceso Constituyente y Nueva Constitución Política convocado por nuestra Facultad de Derecho para contestar las preguntas más controvertidas y relevantes en torno a la demanda por una nueva Constitución para Chile. Se abarcó tanto el mecanismo para arribar a una nueva Constitución como el contenido de la misma y la pregunta sobre si el país necesita una nueva Carta Fundamental.

Sin entrar a describir el contenido que tuvieron las sesiones de discusión, me parece relevante comenzar rescatando esta experiencia, puesto que nos permite enfrentar una pregunta anterior: ¿debe la Universidad de Chile involucrarse en la discusión acerca de una nueva Constitución Política para Chile? Y junto con esto cabe también preguntar: ¿qué tipo de involucramiento debería tener este plantel en una discusión como ésta? En realidad, la pregunta última es aquella vinculada con la relación entre la Universidad y la sociedad; más claramente, el papel que le cabe a una universidad pública frente a las contingencias propias del medio en el que está inserta.

La tensión parece producirse entre quienes pueden considerar que los asuntos debatibles y de alta contingencia no deben ser objeto de involucramiento por parte de la Universidad, la que debería dedicarse exclusivamente al cultivo del saber y la búsqueda de la verdad; y una posición que sostenga que corresponde a la Universidad de Chile derechamente tomar partido respecto de este debate. Entre estos polos de tensión me propongo sostener que la Universidad de Chile, en tanto universidad pública, tiene un deber de participar en debates contingentes de relevancia política para el país. No obstante, de la mano de la idea de universidad pública se sigue que dicha participación no puede ser interesada o "militante", sino que el deber de la Universidad de Chile es el de involucrarse promoviendo una discusión racional y libre.

Para desarrollar esta idea — simple, pero esencial— me referiré, en primer lugar, a la relación entre universidad y sociedad, sosteniendo que de la idea misma de 
universidad se desprende que ésta sea sensible a las coyunturas del medio en el que está inserta, lo que exige un involucramiento activo. En segundo lugar, de la mano del concepto de universidad pública se afirmará que en su rol de conciencia crítica de la sociedad debe la universidad ser un espacio de discusión racional y libre de la injerencia de todo tipo de intereses particulares. A partir de aquí concluiremos que, de cara al proceso constituyente que está teniendo lugar en nuestro país, le corresponde a la Universidad de Chile como un deber involucrarse, promoviendo una discusión profunda, sometiendo a una revisión crítica las razones que circulan en el debate.

\section{UNIVERSIDAD Y SOCIEDAD}

Como se sabe, la universidad moderna ha conocido dos grandes modelos: el modelo francés y el modelo alemán o también llamado humboldtiano. El primero vería la luz en 1806 con la decisión de Napoleón de crear la Universidad Imperial. El segundo, con la influencia que ejerció Wilhelm von Humboldt sobre el rey de Prusia para fundar la Universidad de Berlín sobre bases que se apartaban del modelo napoleónico.

La universidad concebida por Napoleón estaba destinada a la formación de los profesionales que la nación requería para su construcción. Era, además, un modelo centralizado y jerarquizado. Las instituciones de educación superior del modelo francés estaban sujetas a una estricta disciplina, organizadas y controladas por un despotismo ilustrado que gobernaba hasta el último detalle del currículo, la concesión de grados y la adecuación de las visiones académicas con las doctrinas oficiales (Brunner \& Peña, 29-30).

Es común escuchar que éste habría sido el modelo que inspiró a las universidades latinoamericanas. Pero para autores como José Joaquín Brunner y Carlos Peña esta influencia es "más retórica que real", sobre todo en atención a que jamás llega a instalarse en la región un Estado altamente racionalizado, que reclamara identidad de intereses con la ciudadanía y que, en ese contexto, desplegara un sistema de severos controles en la gestión y dirección de la enseñanza universitaria (Brunner \& Peña, 30-31). En el caso de la Universidad de Chile, se sabe que para su fundador y primer Rector, don Andrés Bello, el referente del modelo alemán fue muy importante (Riveros, 20). De todos modos, ciertos rasgos de la recién creada institución hablaban también de una cierta influencia del modelo napoleónico, como el hecho de que el Presidente de la República fuera su patrono, la participación de miembros del gobierno en el Consejo Universitario, la organización de la Universidad a través de facultades con una temprana especialización profesional de las enseñanzas, y una estrecha definición de las carreras. 
El modelo humboldtiano, en cambio, proponía una universidad dedicada al cultivo de un saber superior y la búsqueda de la verdad, relevándose el rol de la producción de conocimiento por sobre la función tradicional de transmisión del mismo. Respecto de este modelo de universidad, al Estado le corresponderían únicamente las tareas de protección de sus libertades y contratación de profesores. Asimismo, la relación entre la universidad y las autoridades estatales debía caracterizarse por la libertad (Brunner \& Peña, 32). Bajo este esquema, se ha dicho que la universidad no debe intervenir en los problemas sociales contingentes y todo su esfuerzo debe trasladarse a la investigación (Novoa, 9).

Para lo que nos interesa relevar, hay algo que tiene el modelo francés que no existe en el modelo humboldtiano. Del mismo modo, hay algo que tiene el modelo humdoldtiano que no tiene el francés. En este último se observa, por una parte, una radicalización del vínculo entre la universidad y las necesidades del país, las que son definidas de manera vertical desde el Estado. Pero como contrapartida existe una ausencia de autonomía del quehacer universitario, es decir, no es libre la universidad para desarrollar el pensamiento y proveer la formación de profesionales, sino que todo esto queda sujeto a la agenda estatal. En el caso del modelo humboldtiano, la importancia de la autonomía respecto de cualquier interés externo es evidente, pero esto viene aparejado de un aislamiento del medio en el que la universidad está inserta. Estos modelos nos hablan, entonces, de una tensión entre la autonomía universitaria, que en su versión extrema supone una desvinculación total con el medio, donde la universidad se aboca de manera libre, pero también exclusiva, a la búsqueda de la verdad y a la producción de conocimiento; y, por otro lado, la necesidad de vincular el quehacer universitario a la contingencia y necesidades locales, que en su versión extrema puede llegar a implicar la intervención del gobierno de turno en la dirección de la universidad.

Jorge Millas, preocupado por el futuro de la universidad chilena durante el periodo de reforma universitaria de los ` 60 , se preguntó por esta tensión. Y para Millas la respuesta estaba revestida de cierta obviedad. Dirá que "[n]o hay, en efecto, programa de reforma universitaria que no comience llamando la atención de la conciencia pública sobre esta cosa tan obvia de que la universidad ha de ser función de su tiempo y de su medio". (Millas, 31). Jorge Millas estaba reaccionando a un movimiento reformista que denunciaba una falta de comunicación entre las universidades y el medio social, pero para él dicho vínculo era evidente, bastaba que se dejase a la universidad cumplir con su misión de cultivo del conocimiento y el resto vendría por añadidura.

Pero lo cierto es que Millas veía en el movimiento reformista un riesgo para el futuro de la universidad. Veía, en definitiva, en las voces que clamaban por una universidad al servicio de las necesidades del país la realización de la posibilidad 
de una desnaturalización de la idea misma de universidad, o lo que él llamaría idea "absoluta" de universidad. Y lo que la universidad no podía dejar de ser, según Millas, es "una comunidad de maestros y discípulos destinada a la transmisión y al progreso del saber superior". (Millas, 34). En esta misma línea y advirtiendo el riesgo que para él era importante denunciar, diría también que la universidad, antes que nada, "tiene que ser el baluarte del conocimiento inspirado en la verdad y en la libertad y regulado por la discusión crítica y si, a pretexto de cumplir otras misiones que se proclamen exigencias de la época, la universidad traiciona ésta, se destruye a sí misma" (Millas, 37).

No obstante, para Millas nada de esto permitía hablar de la universidad en abstracto, "de espaldas" a la realidad de la vida (Millas, 33). Millas sostiene que la universidad pertenece a un medio y a un tiempo, eso es lo que para él resulta obvio. Lo que le preocupa es que eso suponga renunciar a ser universidad. Es en ese sentido que enarbola su idea absoluta de universidad, reivindicando incluso, de algún modo, la metáfora de aquélla como "torre de marfil". Diría Millas, en un pasaje que conviene citar in extenso:

¿La torre de marfil? Sí, claro, la famosa torre donde mataron a Arquímides los romanos que invadieron Sicilia y de donde sacaron a Servet quienes lo llevaron a la hoguera; la torre desde donde Newton pudo ver que una manzana que cae y la luna que gira se mueven conforme a unas mismas leyes; la torre en donde sorprendió a Pavlov la Revolución Rusa y a donde los revolucionarios acudieron a ofrecerle sus respetos; la torre desde donde, justo por ser alta torre se mira más lejos, y donde, por lo mismo, hay más silencio para dialogar mejor; la torre, en fin, que es de este mundo porque la ha edificado la inteligencia del hombre en la historia, que es su historia en este mundo precisamente. A esa torre se ha referido también Félix Martínez en un trabajo que no puede ser ignorado por nadie que quiera pensar desde la mayor altura a que entre nosotros ha sido llevada la reflexión sobre la universidad. Estas son sus palabras: 'Estimo que los críticos que reprochan a las universidades ser torres de marfil en que se incuba un pensamiento ajeno a la realidad, desconocen el hecho fundamental de que el método científico constituye, precisamente, el modo humano de más objetiva aproximación a la realidad, y que, si el enclaustramiento lo favorece, favorece pues, la toma de conocimiento de la situación histórica y natural. La alternativa de enclaustramiento o contacto con la realidad social es falsa y falsifica el planteamiento de los problemas universitarios. El enclaustramiento es uno de los modos egregios de tomar un contacto profundo y amplio con la realidad humana, es el instrumento social de la reflexión y de la previsión larga'. 
Concuerdo absolutamente con este pensamiento. Y no habría que conceder mayor importancia a la frívola y engañosa metáfora, si no fuera que tras ella se enmascara precisamente la antítesis de lo que como símbolo también significa. Porque la emplean como consigna polémica precisamente los que se empeñan en meter la plaza del mercado político dentro de la universidad, esa plaza donde justo por no ser torre de marfil y sí plaza, más se pregona que se razona, y más importa el poder que la ciencia (Millas, 79).

Otro profesor de la Universidad de Chile, desde una vereda opuesta y haciendo eco de las demandas del movimiento reformista, hizo una defensa del vínculo entre universidad y sociedad. Se trata de Eduardo Novoa Monreal, que en 1978 escribió el ensayo "La universidad latinoamericana y el problema social".

Novoa abre su ensayo formulando una serie de interrogantes, entre ellas algunas de total pertinencia para los temas que estamos intentando abordar. Así, se cuestiona: " ¿No atenta contra la concepción más genuina y universal de Universidad el que ella deba ocuparse de materias que fluyen de circunstancias históricas propias de un momento y de un lugar determinados dentro de la vida del hombre?" (Novoa, 6). Junto con esto, se pregunta también: "¿No se arriesga mezclar a la Universidad en peligrosos antagonismos políticos que destruyan su imagen de institución de altos estudios que ha de ser mantenida al margen de partidarismos que dividen, cuando se propone que se comprometa en el problema social?” (Novoa, 6). En una posición que dista bastante de la planteada por Millas, Novoa va a sostener que la universidad debe servir a la sociedad. Lo que se propone demostrar en su ensayo es que "en los países latinoamericanos el verdadero y más importante servicio que la Universidad proporcione a la sociedad ha de ser el estudio sistemático y profundo del grave problema social que los aqueja, la ruptura de la dependencia económica y cultural y la búsqueda de nuevas soluciones" (Novoa, 25).

Frente a las crisis y problemas sociales, así como respecto de las circunstancias materiales en las que viven los países latinoamericanos, Novoa estima que la universidad debe no sólo ser sensible, sino que le asiste un deber de abordarlos. De este modo, dice Novoa:

Todavía más, corresponde a la Universidad alertar a la sociedad desde su alto solio científico, sobre estos problemas y despertar en ella no sólo la conciencia, sino también la voluntad de los cambios. Otra cosa sería eludir el compromiso que tiene contraído con la verdad y con un aspecto muy especial de éste que es la justicia. Solamente la Universidad que entienda que le corresponde provocar en la sociedad una decisión firme de lucha para producir los necesarios cambios sociales, y que esté dispuesta a estimular iniciativas dentro de ese orden, podrá tener la seguridad dentro de América Latina, de que es fiel a su misión (Novoa, 27). 
Por contrapartida, la universidad que renuncia a este deber y se entrega únicamente a la búsqueda de la verdad y producción del conocimiento es, para Novoa, una universidad que contribuye a perpetuar el statu quo social vigente (Novoa, 27).

Se puede ver entonces que mientras a Millas le interesaba la defensa de la idea absoluta de universidad, sosteniendo que su vínculo con el medio es "obvio", Novoa reivindica un rol activo de la universidad en la función crítica que le corresponde ejercer en el contexto social e histórico del que es parte. Y para Novoa, abandonar este deber es contribuir, más bien, derechamente "tomar partido", por la mantención del statu quo.

Las posiciones de quienes fueran dos grandes profesores de nuestra institución nos dan cuenta del problema que en el fondo nos convoca: la pregunta de qué tipo de relación existe o debe existir entre universidad y sociedad. Sin ofrecer una respuesta acabada al problema, considero que la reducción del vínculo entre universidad y sociedad a una "obviedad" resulta elusiva. Efectivamente, creo, la universidad debe tener una posición crítica y activa respecto del medio en el que se encuentra, promoviendo las reflexiones que permitan que la comunidad esté constantemente repensándose y cuestionando las pretendidas verdades que muchas veces la sumen en el inmovilismo. Si la universidad no asume esta labor como un deber de manera activa, es difícil esperar que la cumpla por mera inercia.

En el caso particular de la Universidad de Chile, el deber de involucramiento activo con el medio encuentra también un correlato en su propia historia. En efecto, detrás de la fundación de la Universidad existía la necesidad de que Chile contara con "un ente pensante, un centro de reflexión que permitiera formar el efectivo caudal del pensamiento propio de un país independiente" (Riveros, 18). La Universidad de Chile jugó un rol crucial en la superación del legado colonial y fue de la mayor relevancia también su rol en la construcción de la naciente república. Sus estamentos han sido actores relevantes en prácticamente todas las grandes coyunturas por las que ha atravesado el país, provocando siempre una reflexión crítica de las mismas. Esto ha requerido siempre de una disposición activa a mantener vivo el vínculo entre universidad y sociedad, no bastando esperar que esto venga por añadidura. Incluso el actual Estatuto de la Universidad de Chile (DFL N ${ }^{\circ} 153$ de 1981) en su artículo $3^{\circ}$ establece que la Universidad "se obliga al más completo conocimiento de la realidad nacional y a su desarrollo por medio de la investigación y la creación; postula el desarrollo integral, equilibrado y sostenible del país, aportando a la solución de sus problemas desde la perspectiva universitaria, y propende al bien común y a la formación de una ciudadanía inspirada en valores democráticos, procurando el resguardo y enriquecimiento del acervo cultural nacional y universal". 
Podríamos decir que el único momento en que la Universidad de Chile fue casi neutralizada como centro de reflexión crítica, de cara a la realidad nacional, fue el oscuro periodo de dictadura militar entre 1973 y 1990. Curiosamente, muchos de quienes se opusieron a la reforma universitaria en defensa de lo que consideraban era la esencia de la idea de universidad, no denunciaron con la suficiente energía no sólo la intervención, sino que el directo ataque que la Universidad de Chile, sus académicos y estudiantes, sufrieron durante este periodo. Este triste episodio de la historia de nuestra institución nos muestra que lo que puede presentarse como una posición "neutra" -aquella que alerta sobre los riesgos que para la idea de universidad supone el vínculo entre ésta y la sociedad- puede terminar siendo, en realidad, políticamente conservadora ${ }^{1} \mathrm{o}$, como bien lo planteaba Novoa, partidaria del statu quo.

\section{LA UNIVERSIDAD PÚBLICA}

Parte de la discusión actual ha girado en torno al concepto de universidad pública y universidad estatal. La distinción se ha vuelto especialmente relevante para efectos de discutir el financiamiento público de la educación superior. Sin entrar en este momento en dicho debate, me parece relevante, no obstante, rescatar el concepto de universidad pública para efectos de profundizar en la idea que se está intentando desarrollar. La universidad pública es, a mi parecer, aquella donde ocurre el proceso reflexivo que se hace cargo de la realidad en la que está inserta, debido precisamente a que su estructura organizacional impide que responda a intereses o agendas particulares. De la misma manera, la universidad no debe tomar partido como institución, no debe transformarse en una universidad "militante", sino que

1. Esta es la crítica que el Profesor Fernando Muñoz ha esbozado hacia la idea de universidad de Jorge Millas en una columna titulada "Jorge Millas y la universidad: reflexiones críticas", publicada en el diario El Mostrador el día 25 de marzo de 2014. Muñoz se refiere a la idea de Millas como una "que giraba en torno a una concepción academicista, a primera vista despolitizada pero en última instancia políticamente conservadora, de la labor intelectual y de la transmisión de sus resultados". Muñoz recuerda aquí la actitud original de Millas ante la intervención militar de las universidades. Como lo habría reconocido el mismo Millas, para él la intervención militar apareció en un principio como un mal menor que venía a poner fin a la desnaturalización de la universidad producida por el proceso de reforma. Asimismo, señala Muñoz que si bien Millas se opuso finalmente a la intervención militar de la Universidad de Chile, guardó cómodo silencio con la persecución y exilio (entre los exiliados estuvo el mismo Eduardo Novoa) de sus colegas, silencio que rompió sólo cuando su propia labor académica se volvió imposible. 
debe provocar la discusión racional y libre, defendiendo así su propia naturaleza de pública.

En sus orígenes, la universidad estatal, en el marco del proyecto emancipador e ilustrado del Estado nacional, estuvo llamada a ser "la única capaz de trascender la particularidad de grupos o intereses parciales y expresar, en cambio, la universalidad del proyecto nacional" (Brunner \& Peña, 55). Pero lo cierto es que así como la universidad requiere ser autónoma respecto de intereses particulares, también necesita serlo respecto del Estado y los gobiernos de turno. En efecto, la posibilidad de constituirse como una masa crítica, reflexiva respecto de su medio, requiere también no estar sujeta a la agenda del gobierno. Lo público de la universidad no se define entonces por su carácter estatal, sino que por la autonomía que le permite ser un centro de pensamiento libre y crítico.

Entre nosotros, Fernando Atria ha precisado esta idea de "lo público". Según Atria, lo público "es un espacio en el que como ciudadanos iguales discutimos y decidimos sobre lo que va en el interés de todos, un espacio en el que la apelación al interés particular no tiene fuerza justificatoria" (Atria, 233). Profundizando esta idea agrega que "lo público es el espacio en el que podemos actuar políticamente para cambiar la forma en que la vida común se organiza. Es el espacio donde el solo hecho de que algo sea de determinada manera no es un argumento definitivo para que continúe siéndolo" (Atria, 236). Así, que exista una universidad pública implica para Atria que "habrá una universidad que no responderá a agenda particular alguna" (Atria, 236).

De este modo, la universidad que muchos intentaron construir hasta el golpe militar de 1973 era justamente un espacio de reflexión independiente de cualquier tipo de agenda que se perfilara como centro de reflexión en torno al devenir de la comunidad. Atria, refiriéndose al proyecto de universidad que surge en el Chile de los '60, dice que la universidad "habría de ser la 'conciencia crítica' de la sociedad, es decir, el espacio donde se espera que la reflexión pudiera mirar más allá de las necesidades inmediatas del presente y pensar en el futuro" (Atria, 281). Y para que la universidad posea el carácter de pública, por cierto que se requiere de un determinado régimen institucional, tanto desde el punto de vista de su relación con el entorno como desde el punto de vista de su gobierno interno. Sin entrar en el detalle de este punto, se ha sostenido que no puede haber universidad pública si no se asegura institucionalmente la autonomía universitaria, el financiamiento fuera del mercado y la libertad de académicos y estudiantes para perseguir sus ideas (Atria, 313 y ss.). Respecto del funcionamiento en su interior, no sólo la existencia de órganos colegiados, consultivos y resolutivos, sino que la libre elección de sus autoridades propenden a perseguir los objetivos determinados por su propio ejercicio, sin responder a intereses particulares. 
Lamentablemente, el carácter público de la universidad, incluso el de la Universidad de Chile, se enfrenta a fuertes amenazas. Éstas ponen en peligro, por tanto, la posibilidad de que la Universidad se posicione de frente al momento histórico por el que atraviesa el país. Quizás la principal sea el proceso de profunda mercantilización al que se ha visto sometido el sistema universitario chileno a partir de la década de los '80. En efecto, la proliferación de universidades profesionalizantes y el abandono de las entidades estatales han creado una presión hacia la competencia por estudiantes y el auto-financiamiento de acuerdo a las reglas del mercado. Como lo ha planteado también Atria, "la comunidad de académicos se transforma en una empresa, la conciencia crítica en competencias para el desempeño en el mercado y el conocimiento en algo que importa por su valor de cambio" (Atria, 289).

Una segunda amenaza la encontramos en la abrumadora neutralización de la discusión pública, lo que por cierto resulta muy funcional al proceso de mercantilización. La sociedad chilena, luego de los largos años en que todo tipo de reflexión fue anulado, se ha acostumbrado a la desaparición de los espacios de deliberación. Se ha vuelto costumbre también entregar las grandes decisiones no a la discusión, sino a la negociación de intereses. Así, vemos cómo a veces se toman decisiones con tremendas repercusiones públicas luego de una transacción de votos entre partidos políticos. Es el caso, en mi opinión, de lo ocurrido recientemente durante la tramitación de la llamada "Agenda Corta Antidelincuencia", donde, sin contar con ningún tipo de evidencia y sin hacerse cargo de múltiples advertencias realizadas desde la academia y la sociedad civil, se procedió a aprobar un conjunto de medidas que son de los ejemplos más vergonzosos de populismo penal que hemos conocido en el último tiempo.

En este escenario, la Universidad de Chile mantiene todavía un ethos público, más por esfuerzo de su cuerpo académico y sus estudiantes que por su régimen institucional. En efecto, durante la dictadura militar no sólo se mutiló institucionalmente a la Universidad, sino que se la forzó a ingresar al régimen del mercado y se redujo al mínimo su financiamiento basal, transfiriendo la carga del financiamiento a cada estudiante y sus familias. A pesar de esto, nuestra Casa de Estudios realiza todavía esfuerzos por mantener a flote su misión institucional, cumpliendo en los tres ámbitos que hacen de cualquier institución educativa una universidad: docencia, investigación y extensión. 


\section{LA UNIVERSIDAD DE CHILE FRENTE AL PROCESO CONSTITUYENTE}

Hasta aquí se ha intentado, en primer término, defender una idea de universidad donde se pone de relieve el vínculo entre la universidad y el medio y circunstancias en las que se encuentra inserta. En segundo lugar, hemos sostenido que el concepto de universidad pública exige la autonomía de la entidad universitaria, justamente para que ésta pueda cumplir con su rol de conciencia crítica de la sociedad.

Me interesa relevar estas ideas para fundar el que considero es un deber de nuestra Universidad de involucrarse en el momento histórico por el que atraviesa el país. Chile se encuentra en un proceso de cuestionamiento profundo de las instituciones que rigen nuestra vida en común y el proceso político más relevante, en este escenario, lo representa la deslegitimación de la norma fundamental de nuestra comunidad, la Constitución Política.

El diagnóstico del que me hago parte es aquel que considera que la Constitución de 1980 ha operado como un mecanismo neutralizador de la democracia, perpetuador de una sociedad desintegrada y segregada y que, en definitiva, ha obstado a la construcción de una verdadera comunidad. Los síntomas más claros de este diagnóstico los hemos visto en el descontento ciudadano y la deslegitimación de nuestras principales instituciones.

Considero que tenemos hoy la oportunidad excepcional de reconstruir la convivencia democrática en nuestro país desde la vía institucional, apelando realmente al pueblo para que éste se dé a sí mismo un nuevo arreglo social que le permita autogobernarse, lo que implica reconocer al pueblo como el único titular del poder constituyente. Las consecuencias de esto último son relevantes. No corresponde buscar dentro de la Constitución actual las respuestas para trazar el camino hacia una nueva Constitución, ya que el poder constituyente es anterior al texto constitucional vigente. El poder constituyente es, en palabras de Ernst Böckenförde, "aquella fuerza y autoridad (política) capaz de crear, de sustentar y de cancelar la Constitución en su pretensión normativa de validez" (Böckenförde, 163). Bien señala también Böckenförde que el poder constituyente, como fuerza que hace surgir y legitima la Constitución, no puede existir dentro o sobre la base de la Constitución:

La fuerza que hace surgir y que legitima a la Constitución tiene que representarse por lo tanto, o al menos también, como una magnitud política. Las ideas de justicia y de lo recto, las ideas propias de un orden político, sólo cobran fuerza configuradora y legitimadora para la vida en común de los hombres como una convicción viva, y se integran en una fuerza o en una magnitud política que las sostiene. El poder constituyente (...) [t]iene que entenderse también como una 
magnitud política real que fundamenta la validez normativa de la Constitución. Como tal no puede existir dentro o sobre la base de la Constitución, por ejemplo, como un 'órgano' creado por la Constitución, sino que tiene que preceder a la Constitución y a los pouvoirs contitués nombrados y limitados por ella. Es precisamente esta posición previa y superior frente a los pouvoirs contitués lo que constituye el carácter específico del poder constituyente. (Böckenförde, 163)

Es por esto que no considero posible concebir el ejercicio del poder constituyente a través de los llamados "poderes derivados" o según las actuales reglas constitucionales. Por lo mismo estimo que el mecanismo de la asamblea constituyente es aquel que de mejor forma permite ejercer el poder constituyente del pueblo.

No está demás hacer también un llamado a asumir este proceso sin alarmismo. Desde que la demanda por una nueva Constitución empezó a cobrar fuerza, distintas voces han alertado sobre supuestas nefastas consecuencias que este proceso tendría. Para qué decir sobre la catástrofe que sería entregar a una asamblea constituyente la labor de arribar a un nuevo arreglo social. Todos hemos visto cómo se ha hablado de inestabilidad, de inminente destrucción de la certeza jurídica, de amenaza a derechos fundamentales como la propiedad privada e incluso de las negativas consecuencias que un cambio constitucional tendría para la inversión extranjera. No veo en estas reacciones mucho más que una oposición encubierta a la modificación de las actuales reglas que para algunos han resultado muy beneficiosas. Adicionalmente, este tipo de oposición también habla de un cierto desprecio hacia la democracia y hacia la idea del autogobierno.

En cuanto al papel que le cabe a la Universidad de Chile en este proceso, según lo que hemos dicho le corresponde promover un debate racional e informado, libre de cualquier agenda particular. Asimismo, le compete someter a un análisis crítico todo aquello que se presenta como verdad en el debate, pesar los argumentos, develar la ideología disfrazada de conocimiento experto, y muy importantemente, mostrar si acaso hay peligros donde se dice que los hay. Por lo demás, no cabe duda de que no hay discusión política más relevante que aquella que versa sobre las bases fundamentales de la vida en común. Las posibilidades de que los intereses particulares intenten capturar este debate son altas. Debido a eso mismo se torna crucial aquello que ocurra en la universidad pública en tanto se trata de aquellos espacios que debiesen resguardar la reflexión racional y crítica, resistiendo la injerencia de los intereses particulares.

Como Facultad de Derecho de la Universidad de Chile tuvimos la experiencia durante el año 2015 de promover un ciclo de discusiones en torno al proceso constituyente y la idea de una nueva Constitución Política. Con dicho fin se convocó 
a académicos de las más variadas posiciones. Se buscó siempre conformar mesas de discusión plurales, incentivando el encuentro y el enfrentamiento de ideas. Estas sesiones de discusión tuvieron como resultado la edición de un libro recopilatorio de las distintas ponencias, así como de las preguntas y comentarios de los asistentes. Este libro ha sido difundido abiertamente y fue entregado también a la Presidenta de la República.

Más recientemente, la Facultad de Derecho asumió el llamado a ser sede para la formación de quienes ejercerán como facilitadores de los encuentros locales que tendrán lugar en todo el territorio nacional, de acuerdo al itinerario propuesto por el actual Gobierno. La decisión de que fuera la Universidad de Chile la encargada de esta labor se fundó precisamente en el hecho de que es éste un espacio para la discusión libre de la injerencia de intereses particulares. Incuso las federaciones, tanto de funcionarios como de estudiantes, convocaron a toda la comunidad universitaria a la realización de encuentros locales en la Universidad, actividad que se llevó a cabo satisfactoriamente.

¿Traicionan este tipo de experiencias el quehacer universitario? ¿Desnaturalizan la idea de universidad? ¿Sustituyen al saber por el mercado de la política, como lo denunciara en su momento Jorge Millas? En absoluto. Por el contrario, según lo que hemos expuesto, este tipo de actividades son la forma mediante la cual la Universidad de Chile responde a coyunturas de la mayor relevancia para el país y a la realización misma de la idea de universidad pública. Además, no ha implicado esto que la Universidad de Chile defienda una posición, sino sólo provocar el encuentro de posiciones y la reflexión crítica. Así, en el marco del proceso constituyente, la Universidad de Chile debiese seguir poniendo a disposición sus dependencias para la realización de actividades de discusión; todos sus estamentos deberían sentirse interpelados a contribuir al debate y el quehacer universitario no debiese desatender el deber de ejercer una función crítica respecto del proceso, tanto en lo que respecta a los llamados mecanismos o aspectos formales como los aspectos de fondo, que son, en realidad, parte de un mismo continuo.

\section{CONCLUSIONES}

No hemos pretendido más que ofrecer algunas notas reflexivas en torno a la manera en que nuestra universidad debe vincularse con el medio en el que se encuentra inserta, en concreto, con aquella relevante coyuntura histórica que es el proceso constituyente. Hemos intentado defender la idea según la cual resulta inherente al concepto de universidad la búsqueda activa de una relación entre ésta y el medio en el que se sitúa. Junto con esto, podemos concluir que una universidad pública, 
como espacio libre de intereses particulares, es el lugar propicio para promover una reflexión crítica respecto de coyunturas como lo son el proceso constituyente. Este proceso en particular requiere de una entidad pensante que provoque el enfrentamiento de ideas y desafíe constantemente lo que puedan aparecer en el debate como verdades.

El llamado que estimo debiese interpelarnos a todos quienes formamos parte de la comunidad de la Universidad de Chile es a asumir el involucramiento en el proceso constituyente como un deber, requiriendo de nosotros una posición activa. Junto con esto, igualmente interpelados debiésemos sentirnos respecto de la necesidad de defender a la universidad pública como espacio donde se hace posible la reflexión racional libre de la injerencia de intereses particulares, donde debe tener lugar el pensamiento crítico vinculado al presente y futuro de la comunidad. No debemos olvidar que si nos permitimos ser pasivos ante estos llamados podremos terminar siendo, quizás sin darnos cuenta, partidarios del estado actual de las cosas. 


\section{REFERENCIAS}

Atria, Fernando. Derechos sociales y educación: un nuevo paradigma de lo público. Santiago, LOM, 2014.

Böckenförde, Ernst Wolfgang. Estudios sobre el estado de derecho y la democracia. Madrid, Editorial Trotta, 2000.

Brunner, José Joaquín, Peña, Carlos. La dialéctica público/privado entre el medioevo y la globalización. En: Brunner, José Joaquín, Peña, Carlos. (Eds.). El conflicto de las universidades: entre lo público y lo privado. Santiago, Ediciones Universidad Diego Portales, 2011.

Millas, Jorge. Idea y defensa de la universidad. Santiago, Ediciones Universidad Diego Portales, 2012.

Novoa, Eduardo. La universidad latinoamericana y el problema social. Distrito Federal, Universidad Nacional Autónoma de México, 1978.

Riveros, Luis. Tres siglos venciendo fronteras. El devenir institucional de la Universidad de Chile. Santiago, Ediciones Radio Universidad de Chile, 2016. 